\title{
Modeling Nonlinear Power Amplifiers in OFDM Systems from Subsampled Data: A Comparative Study Using Real Measurements
}

\author{
Ignacio Santamaría \\ Communications Engineering Department (DICOM), University of Cantabria, Avda. Los Castros s/n, 39005, Spain \\ Email: nacho@gtas.dicom.unican.es

\begin{abstract}
Jesús Ibáñez
Communications Engineering Department (DICOM), University of Cantabria, Avda. Los Castros s/n, 39005, Spain Email: jesus@gtas.dicom.unican.es
\end{abstract}

\section{Marcelino Lázaro}

Communications Engineering Department (DICOM), University of Cantabria, Avda. Los Castros s/n, 39005, Spain Email:marce@gtas.dicom.unican.es

\section{Carlos Pantaleón}

Communications Engineering Department (DICOM), University of Cantabria, Avda. Los Castros s/n, 39005, Spain Email:carlos@gtas.dicom.unican.es

\author{
Luis Vielva \\ Communications Engineering Department (DICOM), University of Cantabria, Avda. Los Castros s/n, 39005, Spain \\ Email: luis@gtas.dicom.unican.es
}

Received 19 April 2002 and in revised form 20 February 2003

\begin{abstract}
A comparative study among several nonlinear high-power amplifier (HPA) models using real measurements is carried out. The analysis is focused on specific models for wideband OFDM signals, which are known to be very sensitive to nonlinear distortion. Moreover, unlike conventional techniques, which typically use a single-tone test signal and power measurements, in this study the models are fitted using subsampled time-domain data. The in-band and out-of-band (spectral regrowth) performances of the following models are evaluated and compared: Saleh's model, envelope polynomial model (EPM), Volterra model, the multilayer perceptron (MLP) model, and the smoothed piecewise-linear (SPWL) model. The study shows that the SPWL model provides the best in-band characterization of the HPA. On the other hand, the Volterra model provides a good trade-off between model complexity (number of parameters) and performance.
\end{abstract}

Keywords and phrases: nonlinear modeling, high-power amplifiers, OFDM signals, subsampling techniques.

\section{INTRODUCTION}

Practical high-power amplifiers (HPAs) exhibit nonlinear behavior, which can become dominant unless the HPA is far from its saturation point. Therefore, to have an accurate nonlinear model for the amplifier is a key factor in order to either evaluate the communication system performance by computer simulation or develop compensation techniques to linearize its behavior (using a predistorter, for instance).
Typically, a power amplifier is represented by nonlinear amplitude (AM/AM) and phase (AM/PM) functions in either polar or quadrature form. These AM/AM and AM/PM curves are measured using a single-tone test signal in the center of the band and they are assumed to be frequency independent (memoryless) over the bandwidth of the communications signal. This assumption limits its use to narrowband applications. A widely used model belonging to this type is Saleh's model [1], which represents the AM/AM and AM/PM curves by two-parameter formulas. This model can 
be extended to wideband signals by considering the model parameters as functions of the frequency $[1,2]$. Nevertheless, the model parameters are again fitted using a sweeping single-tone signal and not a wideband input. This fact questions the model's validity for arbitrary wideband signal with high peak-to-average power ratio such as OFDM. On the other hand, single-tone power measurements cannot be used to accurately characterize phenomena such as intermodulation distortion or spectral regrowth.

Despite its practical limitations, Saleh's model, derived from power continuous-wave measurements, is still widely used in the literature to propose and analyze different linearization techniques for wideband systems $[3,4]$. Moreover, the performance of these proposals is typically evaluated by means of computer simulations. Therefore, it is expected that the mismatch between the actual HPA and the assumed model will cause some degradation of these linearization techniques in practice.

Our first claim is that to avoid these drawbacks, the HPA models should be obtained by fitting the input-output time-domain complex envelope of the wideband signal. In the previous years, several methods for time-domain characterization of RF power amplifiers have been proposed $[5,6]$. In general, these techniques sample a demodulated version of the baseband signal, thus requiring up- and downconverter mixers as well as a preamplifier. These devices must be highly linear, otherwise they would introduce additional nonlinear distortion. A solution to remove frequency conversion errors from the measurement system has been proposed in [7]; however, it requires a precise calibration of the converters and the final setup is quite complex.

In this paper, we use subsampling techniques to directly sample the input and output (attenuated if necessary) of the HPA. With the current data acquisition and instrumentation technology, it is possible to use subsampling for low microwave frequency bands ( $\mathrm{L}$ and $\mathrm{C}$ ) at a reasonable cost. Using this measurement setup, it is possible to develop models from subsampled time-domain data.

In this paper, we develop new models for a GaAs MESFET power amplifier working at $1.45 \mathrm{GHz}$. In particular, we concentrate on models specific for OFDM signals, which are known to be extremely sensitive to nonlinear distortion. A number of experiments varying the power and bandwidth of the multicarrier input signal have been performed. Using the acquired data, a comparative study among the following nonlinear models was carried out: Saleh's model [1], envelope polynomial models (EPMs) with memory [8], Volterra models $[9,10]$, the multilayer perceptron (MLP) model $[11,12]$ and the smoothed canonical piecewise linear model [13]. Some conclusions about the memory of the system are also obtained by using an information-theoretic criterion.

The paper is organized as follows. In Section 2, we describe the measurement systems and the discrete-time signal processing carried out to obtain the input-output complex envelope for the HPA. Section 3 briefly describes the main characteristics of the nonlinear models used in this

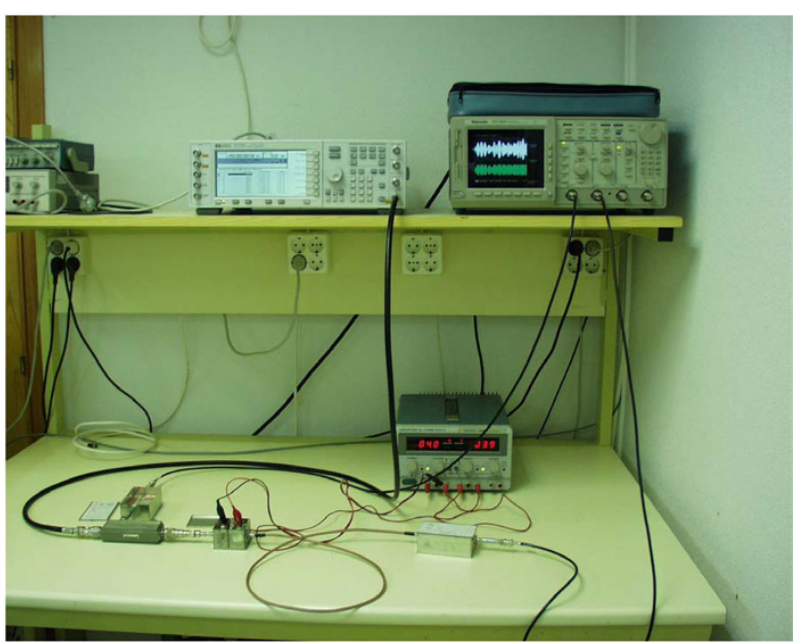

Figure 1: Experimental setup.

study. The performances of these models are compared in Section 4. Finally, the main conclusions are summarized in Section 5.

\section{MEASUREMENT SETUP}

The power amplifier used in this study is a Motorola model MRFC1818 GaAs MESFET. The MRFC1818 is specified for $33 \mathrm{dBm}$ output power with power gain over $30 \mathrm{~dB}$ from a $4.8 \mathrm{~V}$ supply. The used HPA was tuned to provide maximum power at $1.45 \mathrm{GHz}$.

Figures 1 and 2 show the experimental setup and a schematic block diagram of the system, respectively. An $\mathrm{RF}$ signal generator (HP4432B) generates the multicarrier signal; the signal goes through a passband filter tuned to $1.45 \mathrm{GHz}$ and with bandwidth $80 \mathrm{MHz}$; and finally, the input signal is acquired using a digital oscilloscope (Tektronix model TDS694C) which is able to sample up to $10 \mathrm{GHz}$ and store in memory a register of 120000 samples. An exact replica of the acquired input signal, provided by the splitter, is amplified by the HPA under test, bandpass filtered, attenuated (when the signal level is too high), and acquired using the second channel of the oscilloscope.

In this study, OFDM signals with 64 subcarriers were generated using the RF generator HP4432B. Different subcarrier spacing values were considered $\Delta f=45,60,75,90$, $105,120,135$, and $150 \mathrm{kHz}$; in this way, the bandwidth of the OFDM signal ranges from $3 \mathrm{MHz}$ to $10 \mathrm{MHz}$, approximately. Similarly, we carried out the experiments for different input power levels $P_{i}=0,3,6$, and $9 \mathrm{dBm}$, covering from an almost linear amplifier behavior to a strongly saturated point. Finally, we considered different modulation formats for each subcarrier (e.g., BPSK, QPSK, and 64QAM). The main conclusions of this study, however, do not depend on the particular modulation format for each subcarrier.

The processing to acquire the time-domain complex envelope for each experiment is the following. First, the digital oscilloscope acquires the input and output signals using 


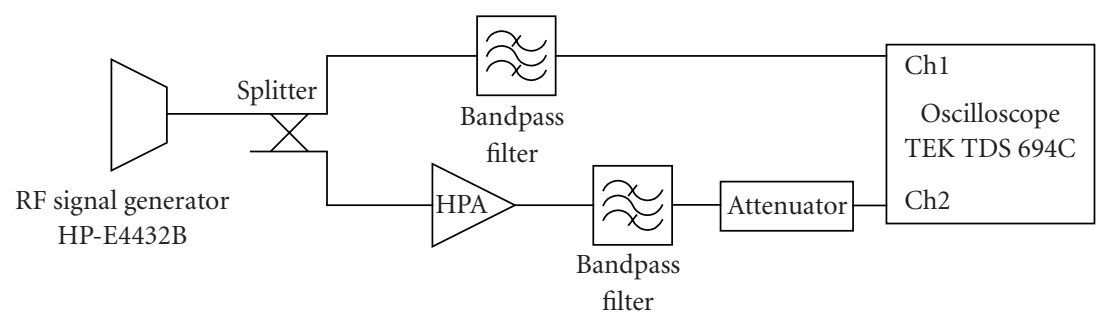

FIgURE 2: Schematic diagram of the measurement system.

a sampling frequency of $1.25 \mathrm{GHz}$. These registers are then transferred via GPIB to a PC. The input and output bandpass OFDM signals, which were originally centered at $1.45 \mathrm{GHz}$, are centered at $1.45 \mathrm{GHz}-1.25 \mathrm{GHz}=200 \mathrm{MHz}$ after the subsampling stage.

Since the passband filters of Figure 2 are not identical, there is some delay between the acquired input and output signals that must be corrected before further processing. This linear delay has been estimated by searching the maximum of the cross-correlation function between the input and output complex envelopes. Note that the delay is estimated at the higher sampling rate (i.e., at $1.25 \mathrm{GHz}$ ), then the uncorrected delay that can be erroneously attributed to the HPA is lower than the sampling period $T=0.8$ nanosecond. Using the estimated linear delay, the input and output complex envelopes are properly time aligned.

Next, the signals are demodulated by the complex exponential sequence $g[n]=e^{-j 2 \pi 0.16 n}$, thus shifting the positive part of the spectrum of the OFDM signals to zero frequency. The complex signals are then lowpass filtered using an FIR filter with 100 coefficients. The specifications of this filter are the following: passband cutoff frequency $=15 \mathrm{MHz}$, transition band $=7 \mathrm{MHz}$, stopband attenuation $=60 \mathrm{~dB}$, and passband ripple $=1 \mathrm{~dB}$. Finally, the signals are downsampled by a factor of 40 , so the final sampling frequency is approximately $31 \mathrm{MHz}$. In this way, the complex envelope of the OFDM signal with the largest bandwidth occupies the band $0-5 \mathrm{MHz}$, and the oversampling ratio is approximately 3 . We consider that this value is enough to characterize the spectral regrowth. With these parameters, the estimated SNR of the input register is approximately $35 \mathrm{~dB}$; this value can be considered as an upper bound on the performance that a perfect HPA model could provide.

The length of the stored registers after downsampling is 3000 samples and we repeat each experiment three times; therefore, for each couple $\left(B W_{i}, P_{i}\right)$, we have 9000 samples of the input-output complex envelope.

An example to highlight the severity of the HPA nonlinear behavior is shown in Figure 3. Here the signal constellation at the output of the FFT processor is plotted for a $6 \mathrm{MHz}$ and $3 \mathrm{dBm} 64 \mathrm{QAM}-\mathrm{OFDM}$ test signal. Unlike single-carrier systems, for which compression and warping effects appear clearly in the constellation, in multicarrier systems, the nonlinear distortion provokes three effects: a phase rotation, a slight warping of the constellation, and, mainly, a distortion

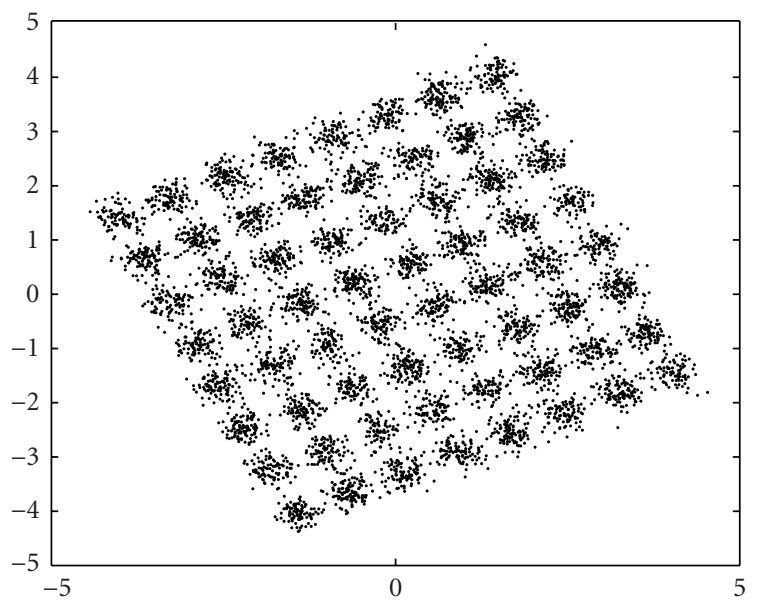

FIGURE 3: Signal constellation at the output of the FFT processor for a 64QAM-OFDM signal with $B W=10 \mathrm{MHz}$ and $P_{i}=3 \mathrm{dBm}$.

that can be modeled as an additive noise. Taking into account that an OFDM signal with a sufficiently large number of carriers can be modeled by a complex Gaussian process with Rayleigh envelope and uniform phase distributions, this nonlinear distortion noise can be theoretically characterized as it is shown in $[14,15]$.

\section{HPA NONLINEAR MODELS}

In this section, we briefly describe the different nonlinear models compared in the study. For each model, we tested polar (modulus/phase) and quadrature (I/Q) configurations. Except for Saleh's model, for which only a polar configuration is considered, the quadrature structure performed slightly better for all the models. For this reason, we will consider only quadrature models.

Probably the most widely known memoryless nonlinear HPA model is Saleh's model, which considers that if the sampled passband input signal is

$$
r[n]=x[n] \cos \left(\omega_{0} n+\phi[n]\right),
$$

then the corresponding output signal is

$$
z[n]=A(x[n]) \cos \left(\omega_{0} n+\phi[n]+\Phi(x[n])\right),
$$




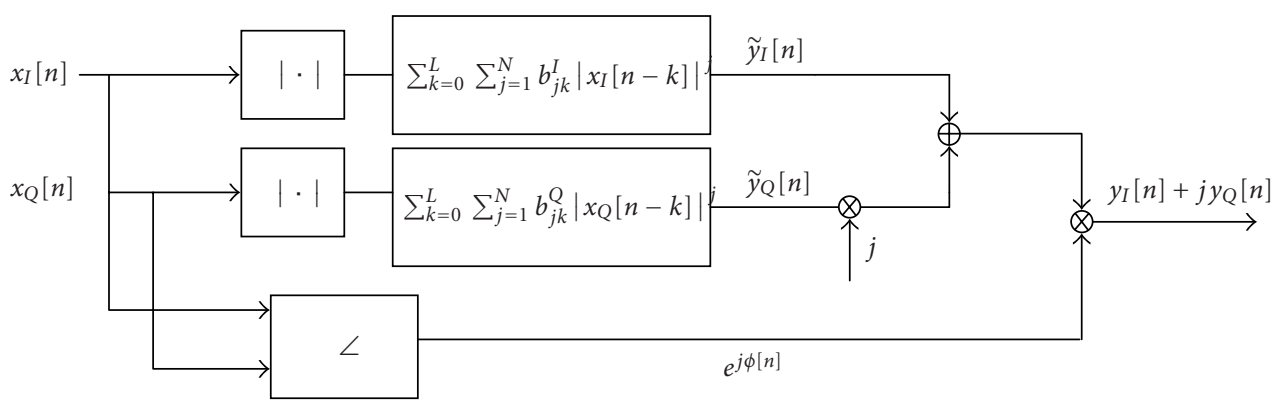

FIgURE 4: Envelope polynomial model.

where the AM/AM and AM/PM curves are given by

$$
\begin{aligned}
& A(x[n])=\frac{\alpha_{a} x[n]}{1+\beta_{a} x[n]^{2}}, \\
& \Phi(x[n])=\frac{\alpha_{\Phi} x[n]}{1+\beta_{\Phi} x[n]^{2}} .
\end{aligned}
$$

Typically, the four parameters of the model are obtained using a single-tone test signal, measuring the amplitude and phase difference, and fitting the curves (3). However, since our goal is to develop specific models for wideband OFDM signals, we have obtained the model parameters by fitting the input-output complex envelope of the subsampled OFDM signal.

In Saleh's model, it is assumed that the characteristics of the HPA are independent of the frequency (memoryless model). In practice, however, when broad-band input signals are involved, a frequency-dependent HPA model is needed. To take into account the memory effects, we use a time delay embedding of the subsampled complex envelope, that is, denoting as $x[n]$ and $y[n]$ the input and output complex envelopes of the wideband OFDM signals, the nonlinear models considered in this paper can be expressed through the following nonlinear mapping:

$$
\left(y_{I}[n], y_{Q}[n]\right)=f\left(x_{I}[n], x_{Q}[n], \ldots, x_{I}[n-d], x_{Q}[n-d]\right) .
$$

The choice of the maximum time delay $d$ plays an important role in the performance of the model (4). This value depends on the particular characteristics of the amplifier, as well as on other factors such as the oversampling ratio of the measurement data set. In this study, we have used the mutual information between the time series $y[n]$ and the delayed time series $x[n-k]$ as an appropriate criterion to estimate the optimum value of the time delay $d$. The time-delayed mutual information was suggested by Fraser and Swinney [16] as a tool to determine a reasonable delay. Unlike the autocorrelation function, the mutual information takes into account also nonlinear correlations. In particular, a detailed analysis that will be described later concluded that the memory of the HPA is just one tap (i.e., any model with memory should use the current and the past sample of the complex envelope). No improvement in performance was achieved by using more than one tap of memory.

The first model with memory is the EPM $[2,8]$ represented in Figure 4. The in-phase and quadrature submodels of order $(L, N)$ have the following input-output relationships:

$$
\begin{aligned}
& \tilde{y}_{I}[n]=\sum_{k=0}^{L} \sum_{j=1}^{N} b_{k j}^{I}\left|x_{I}[n-k]\right|^{j}, \\
& \tilde{y}_{Q}[n]=\sum_{k=0}^{L} \sum_{j=1}^{N} b_{k j}^{Q}\left|x_{Q}[n-k]\right|^{j},
\end{aligned}
$$

where $L$ denotes the memory and $N$ is the highest polynomial order (note that there is not constant term in the polynomial). In the model, the polynomials operate over the modulus of the I/Q components, whereas the phase of the input complex envelope is added at the output. A general study carried out with this model concluded that the best performance was obtained with an $\operatorname{EPM}(1,3)$ with a total number of 12 parameters.

A more general polynomial model with memory is a Volterra series representation of the HPA. In particular, we consider a form of Volterra series suitable to represent bandpass channels [9]:

$$
\begin{gathered}
y[n]=\sum_{k=0}^{M}\left(\frac{\left(\begin{array}{c}
2 k+1 \\
k
\end{array}\right)}{2^{2 k}} \sum_{l_{1}=0}^{L} \ldots \sum_{l_{2 k+1}=0}^{L} h_{2 k+1}\left[l_{1}, \ldots, l_{2 k+1}\right]\right. \\
\left.\times \prod_{r=1}^{k} x^{*}\left[n-l_{r}\right] \prod_{s=k+1}^{2 k+1} x\left[n-l_{s}\right]\right),
\end{gathered}
$$

where $x[n]$ and $y[n]$ denote the input and output complex envelopes, respectively, and $h_{2 k+1}\left[l_{1}, \ldots, l_{2 k+1}\right]$ represent the lowpass equivalent Volterra kernels.

Equation (6) represents a Volterra series expansion of a causal bandpass system for which the terms not lying near the center frequency have been filtered out, and hence have been neglected in the series. The complexity of the Volterra series depends on the number of odd terms in the expansion $1,3, \ldots, 2 M+1$ as well as on its memory $L$ : this model 
is then denoted as Volterra $(2 M+1, L)$. The study carried out with this model concluded that the best performance was obtained with a $\operatorname{Volterra}(3,1)$ model; that is, only the linear and the third-order terms are retained in (6). The total number of parameters in this case is 20 .

The fourth model considered in this study is a conventional MLP whose input-output mapping is given by

$$
\mathbf{y}_{n}=\mathbf{W}_{2}^{T} \tanh \left(\mathbf{W}_{1} \mathbf{x}_{n}+\mathbf{b}_{1}\right)+\mathbf{b}_{2},
$$

where $\mathbf{x}_{n}=\left(x_{I}[n], x_{Q}[n], x_{I}[n-1], x_{Q}[n-1]\right)^{T}$ is the input vector, $\mathbf{y}_{n}=\left(y_{I}[n], y_{Q}[n]\right)^{T}$ is the output, $\mathbf{W}_{1}$ is an $n \times 4$ matrix connecting the input layer with the hidden layer, $\mathbf{b}_{1}$ is an $n \times 1$ vector of biases for the hidden neurons, $\mathbf{W}_{2}$ is an $n \times 2$ matrix of weights connecting the hidden layer to the output neurons, and $\mathbf{b}_{2}$ is an $2 \times 1$ vector of biases for the output neurons. Therefore, we have an $\operatorname{MLP}(4, N, 2)$ structure, where $N$ denotes the number of neurons in the hidden layer. For the MRFC1818 amplifier, the number of neurons to achieve the best performance is $N=10$; then, the total number of parameters of the $\operatorname{MLP}(4,10,2)$ model is 72 . The training of this structure to minimize the mean square error criterion has been carried out using the backpropagation algorithm [17].

Finally, in this study, we consider the SPWL Model [13], which is an extension of the canonical Piecewise-Linear (PWL)model proposed by Chua for microwave device modeling $[18,19]$. In its basic formulation, the canonical PWL performs the following mapping:

$$
\mathbf{y}_{n}=\mathbf{a}+\mathbf{B} \mathbf{x}_{n}+\sum_{i=1}^{N} \mathbf{c}_{i}\left|\left\langle\alpha_{i}, \mathbf{x}_{n}\right\rangle-\beta_{i}\right|,
$$

where $\mathbf{a}$ and $\mathbf{c}_{i}$ are $2 \times 1$ vectors, $\boldsymbol{\alpha}_{i}$ is a $4 \times 1$ vector, $\mathbf{B}$ is a $2 \times 4$ matrix, $\beta_{i}$ is a scalar, and $\langle\cdot, \cdot\rangle$ denotes inner product.

The PWL model divides the input space into different regions limited by hyperplanes, and in each region, the function is composed by a linear combination of hyperplanes. The expression inside the absolute value defines the boundaries partitioning the input space.

The main drawback of the PWL model is that, like the absolute value function, is not derivable. The SPWL model overcomes this lack of derivability by smoothing the boundaries among hyperplanes using the function

$$
\operatorname{lch}(x, \gamma)=\frac{1}{\gamma} \ln (\cosh (\gamma x)),
$$

where $\gamma$ is a parameter controlling the smoothness of the model. Thus, the $\operatorname{SPWL}(4, N, 2)$ model with $N$ boundaries performs the following mapping:

$$
\mathbf{y}_{n}=\mathbf{a}+\mathbf{B} \mathbf{x}_{n}+\sum_{i=1}^{N} \mathbf{c}_{i} l \operatorname{lch}\left(\left\langle\alpha_{i}, \mathbf{x}_{n}\right\rangle-\beta_{i}, \gamma\right)
$$

In this model, we have used $N=10$ boundaries for a total number of 71 parameters.
The SPWL has three different kinds of parameters: those defining the boundaries partitioning the input space: $\boldsymbol{\alpha}_{i}$ and $\beta_{i}$; those defining the linear combination of the model components: $\mathbf{a}, \mathbf{B}$, and $\mathbf{c}_{i}$; and the smoothing parameter $\gamma$. The training algorithm for the SPWL model is an iterative algorithm based on the successive adaptation of the boundaries and the estimate of the optimal coefficients for that given partition. The adaptation of the parameters defining the boundaries in the input space is based on a second-order gradient method. Once the boundaries are fixed, the MSE is a quadratic function of the parameters defining the linear combination of the components, and the minimum can be easily found by solving a linear least squares problem. Then, the boundaries are adapted again and the process is repeated iteratively. On the other hand, the smoothness parameter $\gamma$ is typically a value fixed in advance. More details of this algorithm can be found in $[13,18]$.

\section{EXPERIMENTAL RESULTS}

In this section, we first draw some conclusions about the required memory (maximum time delay) of the nonlinear models. Then we compare the performance of the previously described nonlinear models. Throughout this section, we use QPSK-OFDM and BPSK-OFDM wideband signals. However, we have found that the main conclusions do not depend on the particular modulation format on each subcarrier.

\subsection{Data set analysis}

The training and testing sets are formed from the subsampled time-domain measurements as follows: for each bandwidth and input power, we have 9000 samples of the inputoutput complex envelope; 3000 samples are retained for training the models and 6000 for testing. We have carried out measurements for 8 different bandwidths $3,4,5,6,7,8,9$, and $10 \mathrm{MHz}$, and for four different input powers $P_{i}=0,3,6$, and $9 \mathrm{dBm}$. Therefore, the final training and testing sets for each input power are composed of 24000 and 48000 complex samples, respectively. Our aim is to obtain a different model, independent of the bandwidth, for each input power.

As discussed in Section 3, the choice of the maximum delay $d$ of the time embedding (i.e., the memory) plays an important role in the performance of the HPA model. Assuming that the number of carriers is sufficiently large, the OFDM signal can be modeled by a complex Gaussian process with independent I/Q components. For this reason, here we consider the simpler problem of estimating the optimum value of $d$ for the mapping $y_{I}[n]=f\left(x_{I}[n], \ldots, x_{I}[n-d]\right)$; the conclusions can be readily extended to the global nonlinear model (4). To this end we use an information-theoretic criterion; specifically, we estimate the mutual information between the output time series $y_{I}[n]$ and the delayed input time series $x_{I}[n-k]$ : a value of the mutual information close to zero indicates that there is not any statistical relationship between the two time series. This criterion has been previously used to estimate the dimensionality of dynamical systems from experimental time series $[16,20]$. 
For two random variables $Y$ and $X$, mutual information can be estimated using the Kullback-Leibler (KL) divergence between the joint probability density function (pdf) and the factored marginals, that is,

$$
I_{\mathrm{KL}}(Y, X)=\iint f_{Y X}(y, x) \log \frac{f_{Y X}(y, x)}{f_{Y}(y) f_{X}(x)} d y d x .
$$

The mutual information is a natural measure of the dependence between random variables. It is always nonnegative, and is zero if and only if the variables are statistically independent. Thus the mutual information takes into account the whole dependence structure of the variables. The problem with mutual information is that it is difficult to estimate from data. To solve this problem in this study, we have used the following alternative information-theoretic distance measure

$$
\begin{aligned}
& I_{\mathrm{QMI}}(Y, X) \\
& \quad=\log \frac{\left(\iint f_{Y X}(y, x)^{2} d y d x\right)\left(\iint f_{Y}(y)^{2} f_{X}(x)^{2} d y d x\right)}{\left(\iint f_{Y X}(y, x) f_{Y}(y) f_{X}(x) d y d x\right)^{2}},
\end{aligned}
$$

which is denoted as quadratic mutual information (QMI) and was proposed in $[21,22]$. It can be viewed as a generalized correlation coefficient that estimates the angle between the joint pdf and the product of the marginals. If we estimate the joint pdf and both marginals using the Parzen window method with Gaussian kernels, then the QMI can be easily evaluated: this is the main advantage of (12) in comparison to (11). The details of the estimation procedure can be found in $[21,22]$.

Our aim here is to quantify the amount of "new" information that $x_{I}[n-k]$ provides about $y_{I}[n]$. Therefore, before estimating the mutual information between $x_{I}[n-k]$ and $y_{I}[n]$, we must subtract somehow the information already provided by the previous inputs $x_{I}[n], \ldots, x_{I}[n-k+1]$. Specifically, the applied preprocessing step consists of calculating the mutual information between the delayed time series $x_{I}[n-k]$ and the residual after linear prediction $e[n]=$ $y_{I}[n]-\sum_{l=0}^{k-1} a_{l} x_{I}[n-l]$, for $k \geq 1$. In this way, we eliminate any statistical linear relationship between $y_{I}[n]$ and the previous inputs $x_{I}[n], \ldots, x_{I}[n-k+1]$.

Figure 5 shows the results obtained for a QPSK-OFDM signal with $P_{i}=9 \mathrm{dBm}$ and different bandwidths. For $k \geq 2$, the mutual information between $y_{I}[n]$ and $x_{I}[n-k]$ is practically zero, so both time series can be considered as statistically independent. The conclusion of this analysis is that all the information about $y[n]$ can be extracted from $x[n]$ and $x[n-1]$ (i.e., the memory of the nonlinear models is one tap).

\subsection{A comparative study}

To have a first qualitative idea about the capabilities of the obtained models, Figure 6 compares the measured and estimated power spectral densities (PSDs) at the output of the HPA. In this example, the input signal is a QPSK-OFDM with bandwidth $6 \mathrm{MHz}$ and input power $3 \mathrm{dBm}$ (represent-

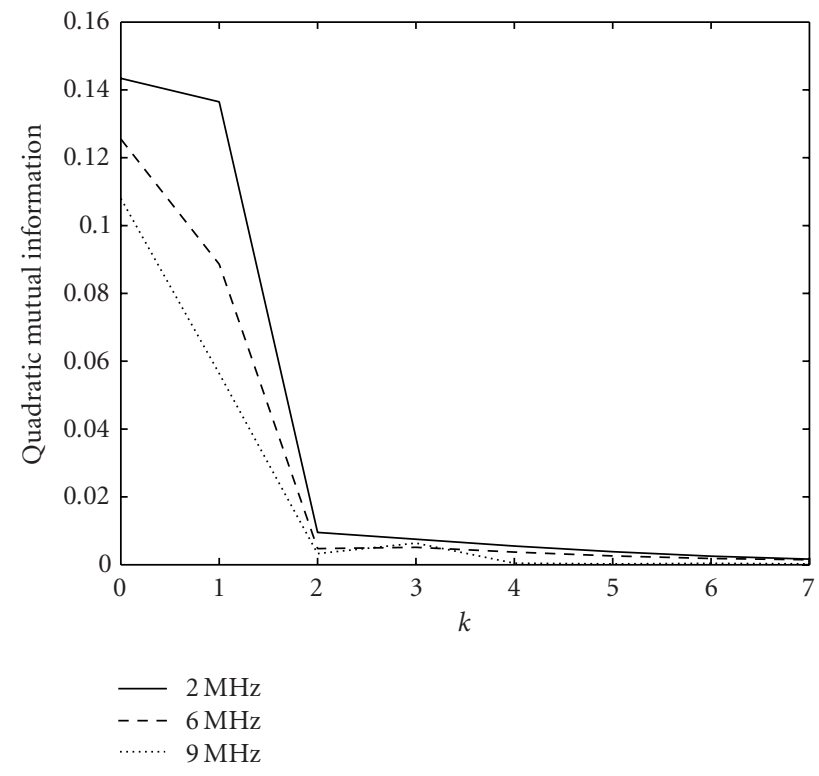

FIGURE 5: Quadratic mutual information between $y_{I}[n]$ and $x_{I}[n-$ $k]$ as a function of $k$ for a QPSK-OFDM signal with $P_{i}=9 \mathrm{dBm}$ and different bandwidths.

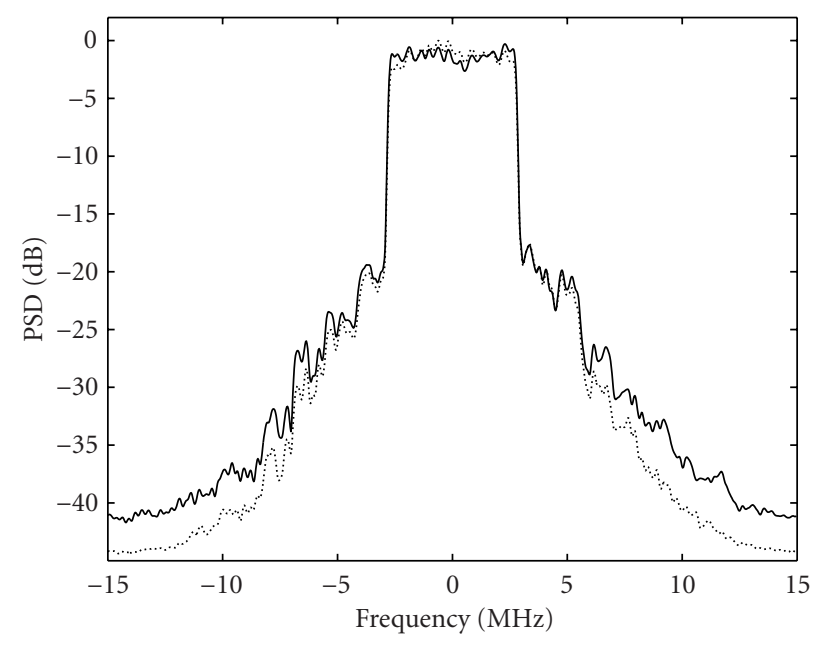

FIGURE 6: Measured (dotted line) and estimated (solid line) PSDs at the output of the HPA. The signal is a QPSK-OFDM with $6 \mathrm{MHz}$ of bandwidth and $3 \mathrm{dBm}$ of input power. The nonlinear HPA model is an MLP.

ing a mild nonlinear behavior), and the nonlinear HPA model is an MLP. The spectral regrowth of the HPA is evident, indicating its nonlinear behavior. On the other hand, we observe a good fitting between the measured PSD and the output of the MLP model. However, the out-of-band distortion at frequencies far from the signal bandwidth tends to be slightly overestimated. This good agreement between the measurements and the estimated signals can be also observed in the time domain (see Figure 7). For higher bandwidths or higher input powers, the performance of the models tends to degrade, as we will show in the following examples. 


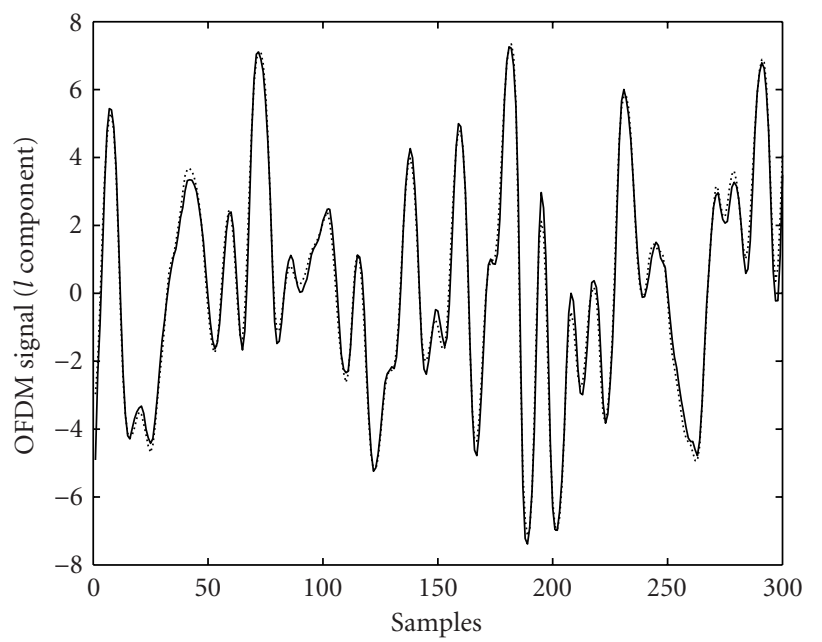

FIgURE 7: Measured (dotted line) and estimated (solid line) time domain signals at the output of the HPA. The signal is a QPSKOFDM with $6 \mathrm{MHz}$ of bandwidth and $3 \mathrm{dBm}$ of input power.

To carry out a more detailed comparative study among the different nonlinear models discussed in this paper, we have considered a BPSK-OFDM signal. A figure of merit, which captures the in-band behavior of the model, is the signal-to-error ratio (SER) defined as

$$
\mathrm{SER}=10 \log \left(\frac{\sum_{n}|y[n]|^{2}}{\sum_{n}|\hat{y}[n]-y[n]|^{2}}\right),
$$

where $\hat{y}[n]$ is the output of the model and $y[n]$ is the actual output of the HPA.

Another figure of merit, specific to evaluate the out-ofband behavior of the HPA, is the adjacent channel power ratio (ACPR). It is defined as the ratio between the power in the input signal bandwidth and the power in either the upper or lower adjacent channels. In this study, we use the mean of the power between the lower and upper channels; specifically, the ACPR is defined as

$$
\operatorname{ACPR}(S(f))=10 \log \left(\frac{2 \int_{-B / 2}^{B / 2} S(f) d f}{\int_{-3 B / 2}^{-B / 2} S(f) d f+\int_{B / 2}^{3 B / 2} S(f) d f}\right),
$$

where $B$ is the bandwidth of the input signal and $S(f)$ is the PSD of the acquired signal (over a bandwidth of $30 \mathrm{MHz}$ ). In order to evaluate the ability of the nonlinear HPA models to reproduce the ACPR, we will use

$$
\triangle \mathrm{ACPR}=\operatorname{ACPR}(\hat{S}(f))-\operatorname{ACPR}(S(f)),
$$

where $\hat{S}(f)$ is the PSD of the output provided by the model and $S(f)$ is the true output.

Figures 8 and 9 compare the SER obtained with the five nonlinear models under test for an input power $P_{i}=0 \mathrm{dBm}$ (slightly nonlinear behavior) and $P_{i}=9 \mathrm{dBm}$ (strongly non-

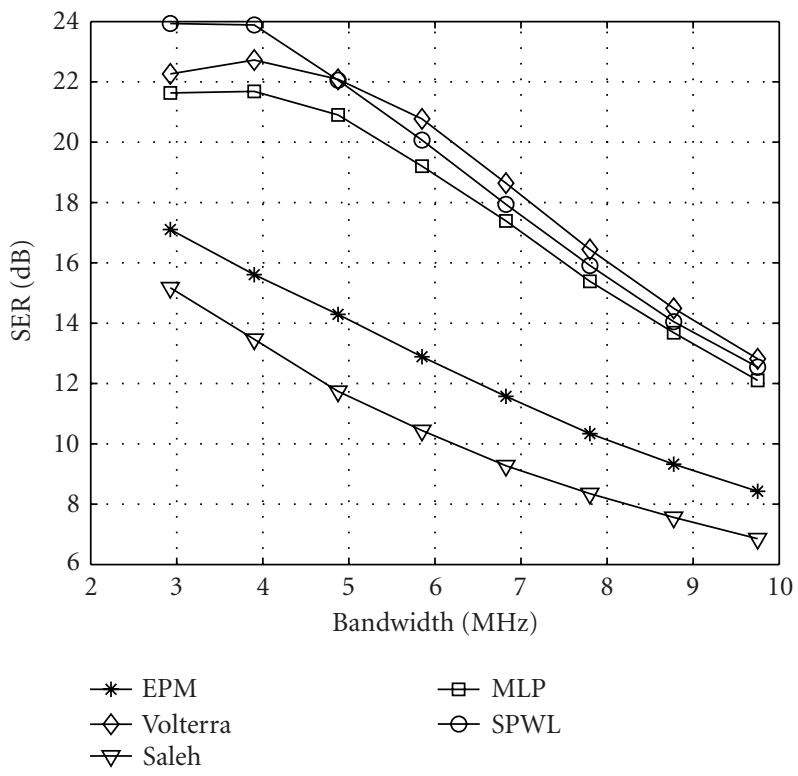

FIGURE 8: SER for an input power $P_{i}=0 \mathrm{dBm}$.

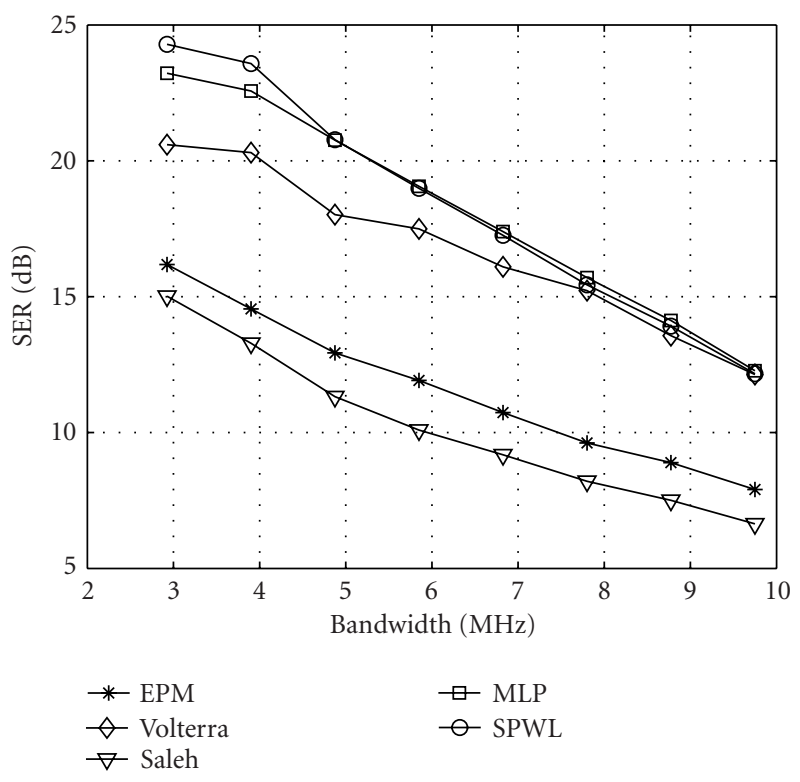

FIGURE 9: SER for an input power $P_{i}=9 \mathrm{dBm}$.

linear behavior), respectively. We can see that, as long as the bandwidth increases, the performance of all the methods decreases. The explanation of the fact is twofold. First, it is clear that keeping fixed the number of model parameters, it is more difficult to adjust a larger bandwidth. Secondly, for larger bandwidths, the distortion due to aliasing increases. On the other hand, Saleh's model and the EPM, with only 4 and 12 parameters, respectively, obviously provide worse results than the MLP, SPWL, and Volterra models, which have a higher number of parameters (70, 71, and 20, resp.). Finally, we can conclude that when the HPA is working far from its 


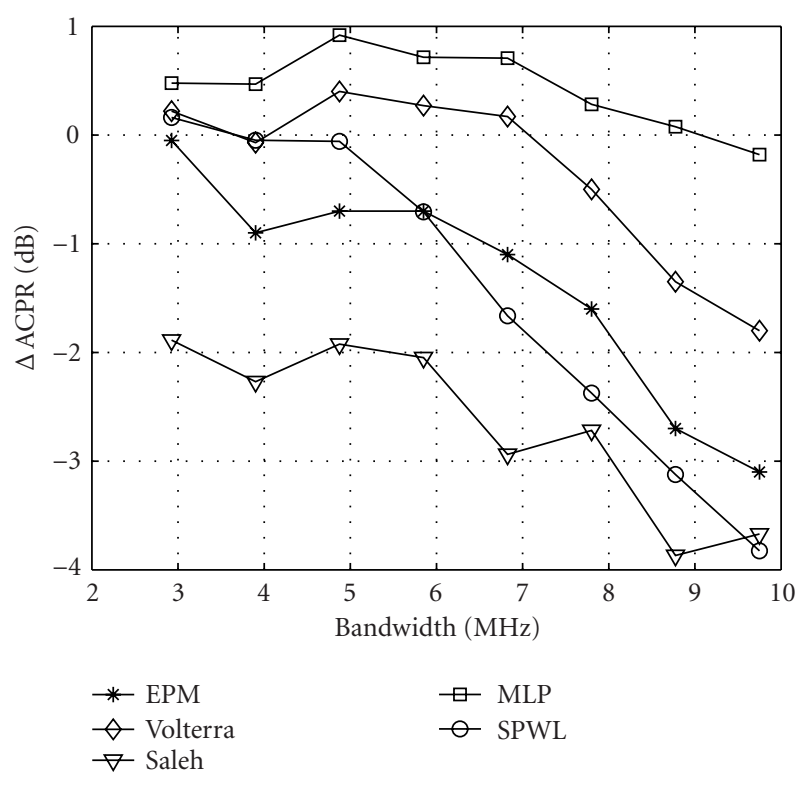

Figure 10: $\triangle \mathrm{ACPR}$ for an input power $P_{i}=0 \mathrm{dBm}$.

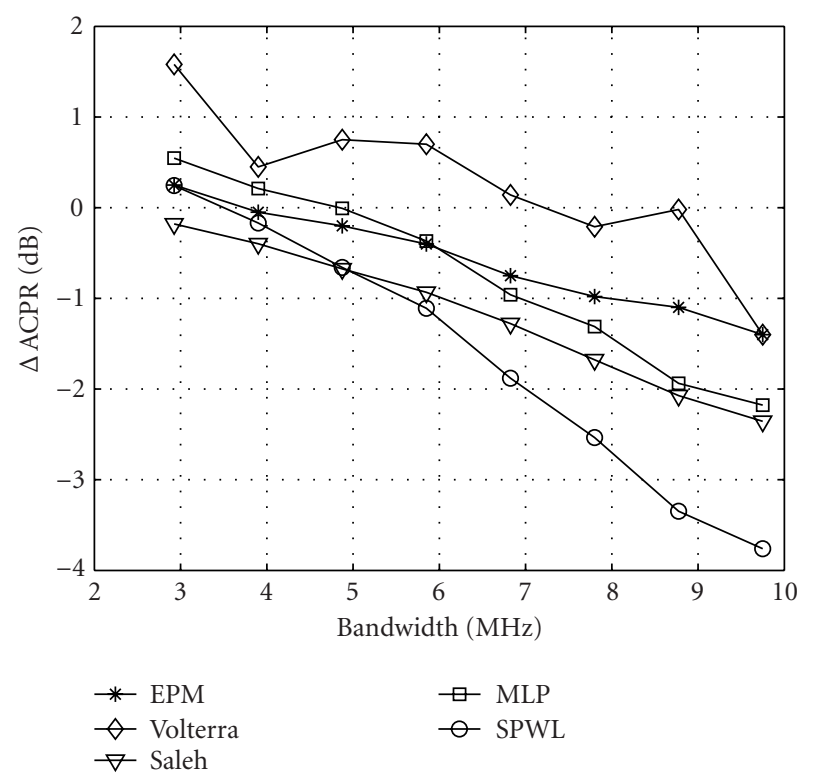

Figure 11: $\triangle \mathrm{ACPR}$ for an input power $P_{i}=9 \mathrm{dBm}$.

saturation point, the best results are provided by the SPWL and the Volterra models.

When the input power increases and the HPA works close to its saturation point, the two neural-based models, that is, the MLP and the SPWL, provide the best results. On the other hand, the performance of the Volterra model degrades, specially for the smaller bandwidths. This degradation of Volterra models for hard nonlinearities is due to the fact that when the input level tends to infinity, the output of any polynomial model also tends to infinity. Therefore, it is not possible to accurately model hard clipping effects with polynomial
TABLE 1: Mean absolute error in the ACPR (in dB).

\begin{tabular}{lccccc}
\hline & $0 \mathrm{dBm}$ & $3 \mathrm{dBm}$ & $6 \mathrm{dBm}$ & $9 \mathrm{dBm}$ & Mean \\
\hline EPM & 1.1200 & 0.5660 & 0.5490 & 0.5710 & 0.7015 \\
Volterra & 0.5980 & 0.6130 & 0.6090 & 0.7030 & 0.6307 \\
Saleh & 2.3981 & 2.1474 & 1.7872 & 0.9990 & 1.8329 \\
MLP & 0.4283 & 0.4788 & 0.4364 & 0.8649 & 0.5521 \\
SPWL & 1.2121 & 1.3673 & 1.2230 & 1.4753 & 1.3194 \\
\hline
\end{tabular}

models, which is an important drawback of Volterra models (and EPMs).

To evaluate the out-of-band behavior, that is, the capacity of modeling the spectral regrowth, Figures 10 and 11 show the $\triangle$ ACPR obtained with each model for $P_{i}=0$ and $9 \mathrm{dBm}$, respectively. In these figures, a value of $\triangle \mathrm{ACPR}=0$ means a perfect match between the out-of-band power of the HPA and the model output. On the other hand, Table 1 shows the mean of $|\triangle \mathrm{ACPR}|$ for each input power.

It is interesting to highlight the following points. Despite its relatively high number of parameters and its good inband performance, the SPWL models tend to underestimate the ACPR, mainly for the larger bandwidths. This means that the spectral regrowth caused by the nonlinear model is larger than the actual one. The same behavior is observed for Saleh's model. Considering the results obtained for all the input powers (see Table 1), the MLP provides the best results.

Finally, considering both the SER and ACPR results, the Volterra model, with only 20 parameters, is a good trade-off between complexity and performance, at least for mild nonlinearities.

\section{CONCLUSIONS}

In this paper, the characteristics of five nonlinear HPA models have been compared with respect to their in-band and out-of-band performances. The comparative study has been carried out using measurements obtained from a GaAs MESFET amplifier, and it has been focused on wideband OFDM signals. For this kind of signals, conventional models obtained using a single-tone test signal are inadequate and better models are obtained by directly fitting the input-output time-domain complex envelope of the OFDM signal. Considering the SER, the best results are provided by the SPWL model, whereas in terms of ACPR, the MLP model gives the best approximation. This result suggests that different models (or different training criteria) should be used depending on whether the aim is to model the in-band or the spectral regrowth behavior. As a final conclusion, we can remark that the Volterra model provides a good trade-off between model complexity (number of parameters) and performance for mild nonlinearities.

\section{ACKNOWLEDGMENT}

This work was partially supported by the European Community and the Spanish MCYT under Projects 1FD97-1863C02-01 and TIC2001-0751-C04-03 (PLASOFTRA). 


\section{REFERENCES}

[1] A. A. M. Saleh, "Frequency-independent and frequencydependent nonlinear models of TWT amplifiers," IEEE Trans. Communications, vol. 29, no. 11, pp. 1715-1720, 1981.

[2] M. C. Jeruchim, P. Balaban, and K. S. Shanmugan, Simulation of Communication Systems, Plenum Press, NY, USA, 1992.

[3] F. J. González-Serrano, J. J. Murillo-Fuentes, and A. ArtésRodríguez, "GCMAC-based predistortion for digital modulations," IEEE Trans. Communications, vol. 49, no. 9, pp. 16791689, 2001.

[4] A. N. D'Andrea, V. Lottici, and R. Reggiannini, "Nonlinear predistortion of OFDM signals over frequency-selective fading channels," IEEE Trans. Communications, vol. 49, no. 5, pp. 837-843, 2001.

[5] M. S. Heutmaker, "The error vector and power amplifier distortion," in Proc. IEEE Wireless Communications Conference (WCC '97), pp. 100-104, Boulder, Colo, USA, August 1997.

[6] V. Borich, J.-H Jong, J. East, and W. E. Stark, "Nonlinear effects of power amplification on multicarrier spread spectrum systems," in Proc. IEEE MTT-S International Microwave Symposium Digest (IMS '98), pp. 323-326, Baltimore, Md, USA, June 1998.

[7] C. J. Clark, G. Chrisikos, M. S. Muha, A. A. Moulthrop, and C. P. Silva, "Time-domain envelope measurement technique with application to wideband power amplifier modeling," IEEE Transactions on Microwave Theory and Techniques, vol. 46, no. 12, pp. 2351-2540, 1998.

[8] J. Ibáñez Díaz, C. Pantaleón, I. Santamaría, T. Fernández, and D. Martínez, "Nonlinearity estimation in power amplifiers based on subsampled temporal data," IEEE Trans. Instrumentation and Measurement, vol. 50, no. 4, pp. 882-887, 2001.

[9] S. Benedetto, E. Biglieri, and R. Daffara, "Modeling and performance evaluation of nonlinear satellite links-A Volterra series approach," IEEE Transactions on Aerospace and Electronic Systems, vol. 15, no. 4, pp. 494-507, 1979.

[10] E. Biglieri, S. Barberis, and M. Catena, "Analysis and compensation of nonlinearities in digital transmission systems," IEEE Journal on Selected Areas in Communications, vol. 6, no. 1, pp. 42-51, 1988.

[11] M. Ibnkahla, J. Sombrin, F. Castanié, and N. J. Bershad, "Neural networks for modeling nonlinear memoryless communication channels," IEEE Trans. Communications, vol. 45, no. 7, pp. 768-771, 1997.

[12] M. Ibnkahla, N. J. Bershad, J. Sombrin, and F. Castanié, "Neural network modeling and identification of nonlinear channels with memory: algorithms, applications, and analytic models," IEEE Trans. Signal Processing, vol. 46, no. 5, pp. 1208-1220, 1998.

[13] M. Lázaro, I. Santamaría, C. Pantaleón, A. Mediavilla, A. Tazón, and T. Fernández, "Smoothing the canonical piecewise-linear model: an efficient and derivable large-signal model for MESFET/HEMT transistors," IEEE Trans. Circuits and Systems I: Fundamental Theory and Applications, vol. 48, no. 2, pp. 184-192, 2001.

[14] D. Dardari, V. Tralli, and A. Vaccari, "A theoretical characterization of nonlinear distortion effects in OFDM systems," IEEE Trans. Communications, vol. 48, no. 10, pp. 1755-1764, 2000.

[15] P. Banelli and S. Cacopardi, "Theoretical analysis and performance of OFDM signals in nonlinear AWGN channels," IEEE Trans. Communications, vol. 48, no. 3, pp. 430-441, 2000.

[16] A. M. Fraser and H. L. Swinney, "Independent coordinates for strange attractors from mutual information," Physical Review A, vol. 33, no. 2, pp. 1134-1140, 1986.
[17] S. Haykin, Neural Networks: A Comprehensive Foundation, Macmillan Publishing Company, NY, USA, 1998.

[18] L. O. Chua and A. C. Deng, "Canonical piecewise-linear modeling," IEEE Trans. Circuits and Systems, vol. 33, no. 5, pp. 511-525, 1986.

[19] L. O. Chua and A. C. Deng, "Canonical piecewise-linear representation," IEEE Trans. Circuits and Systems, vol. 35, no. 1, pp. 101-111, 1988.

[20] F. J. Pineda and J. C. Sommerer, "Estimating generalized dimensions and choosing time delays: a fast algorithm," in Time Series Prediction: Forecasting the Future and Understanding the Past, A. S. Weigend and N. A. Gershenfeld, Eds., vol. 15 of SFI Studies in the Sciences of Complexity, pp. 367-385, AddisonWesley, Reading, Mass, USA, 1993.

[21] J. C. Principe, D. Xu, Q. Zhao, and J. W. Fisher III, "Learning from examples with information theoretic criteria," Journal of VLSI Signal Processing-Systems, vol. 26, no. 1-2, pp. 61-77, 2000.

[22] J. C. Principe, D. Xu, and J. W. Fisher III, "Informationtheoretic learning," in Unsupervised Adaptive Filtering, S. Haykin, Ed., pp. 265-319, John Wiley \& Sons, NY, USA, 1999.

Ignacio Santamaría was born in Vitoria, Spain in 1967. He received his Telecommunication Engineer degree and his Ph.D. degree in electrical engineering from the Polytechnic University of Madrid, Spain in 1991 and 1995, respectively. In 1992, he joined the Departamento de Ingeniería de Comunicaciones at the Universidad de Cantabria, Spain, where he is currently an Associate Professor. In 2000, he spent a visiting period at the Computational NeuroEngineering Laboratory (CNEL), University of Florida. Dr. Santamaría has more than 60 publications in refereed journals and international conference papers. His current research interests include nonlinear modeling techniques, adaptive systems, and machine learning theories and their application to digital communication systems.

Jesús Ibáñez was born in Santander, Spain in 1971. He received the radiocommunication B.S. degree in engineering and the Telecommunication Engineer degree from the Universidad de Cantabria, Spain in 1992 and 1995, respectively. In 1995, he joined the Departamento de Ingeniería de Comunicaciones at the Universidad de Cantabria, Spain, where he is currently an Associate Professor. His research interests include digital signal processing, digital communication systems, and nonlinear systems.

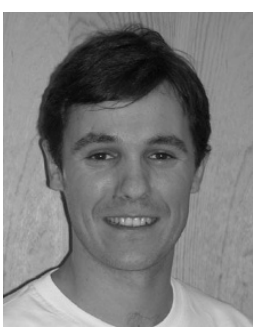

Marcelino Lázaro was born in Carriazo, Spain in 1972. He received the Telecommunication Engineer degree and the Ph.D. degree from the Universidad de Cantabria, Spain in 1996 and 2001, respectively. From 1996 to 2002, he worked in the Departamento de Ingeniería de Comunicaciones at the Universidad de Cantabria, Spain. In 2003, he joined the Departamento de Teoría de la Señal y Comunicaciones at the Universidad Carlos III de Madrid. His research interests include digital

signal processing, nonlinear modeling and neural networks.

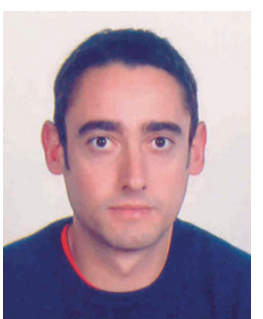


Carlos Pantaleón was born in Badajoz, Spain in 1966. He received the Telecommunication Engineer degree and the Ph.D. degree from the Universidad Politécnica de Madrid (UPM), Spain in 1990 and 1994, respectively. In 1990, he joined the Departamento de Ingeniería de Comunicaciones at the Universidad de Cantabria, Spain, where he is currently an Associate Professor. His research interests include digital signal pro-

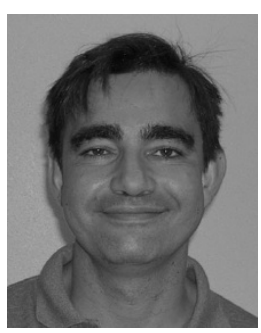
cessing and nonlinear and chaotic systems.

Luis Vielva was born in Santander, Spain in 1966. He received his Licenciado degree and his Ph.D. degree in physics from the Universidad de Cantabria, Spain in 1989 and 1997, respectively. In 1989, he joined the Departamento de Ingeniería de Comunicaciones, Universidad de Cantabria, Spain, where he is currently an Associate Professor. In 2001, he spent a visiting period at the Computational NeuroEngineering Labora-

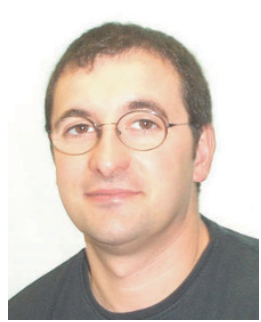
tory (CNEL), University of Florida. Dr. Vielva has more than 50 publications in refereed journals and international conference papers. His current research interests include blind source separation and bioinformatics. 